\title{
Gynæcologic Symptoms in Patients with Non-cœliac Wheat Sensitivity
}

\author{
Sophie W. Hall ${ }^{1,3} \cdot$ Andrew S. Day ${ }^{2,3}$
}

Published online: 14 May 2020

(c) Springer Science+Business Media, LLC, part of Springer Nature 2020

With increasing interest in recent years in cœliac disease (CD) and also in alternate clinical presentations related to dietary exposure to components of cereals, there has been consequent increasing popularity of gluten-free diets (GFD). Increasing rates of $\mathrm{CD}$ have been documented in several settings [1]. However, even greater numbers of individuals have adopted a GFD for a range of other reasons [2], generating huge increases in the market for GF products [3]. Within this spectrum of cereal-related disorders is non-cœliac gluten sensitivity (NCGS), also termed non-coliac wheat sensitivity (NCWS) [4].

NCGS/NCWS was defined in 2015 as an immune-mediated reaction to wheat manifest as several gastrointestinal (GI) or non-gastrointestinal symptoms that improve with the exclusion of gluten or wheat, in a setting where CD and wheat allergy have both been excluded [5]. Withdrawal of gluten or wheat from the diet followed by a direct challenge is regarded as the gold standard, with a double-blind placebo-controlled (DBPC) re-challenge trial considered optimal in research settings. At present, and unlike $\mathrm{CD}$, there are no laboratory assays such as biomarkers or histological findings that provide accurate and objective indicators for this condition. Consequently, strict use of standardized diagnostic criteria is essential. Furthermore, controversy remains as to the contribution of other specific cereal components such as fructans (one of the fermentable carbohydrates, a component of the FODMAPs group) or amylase/trypsin inhibitors $[4,6]$. This further highlights the importance of a well-defined and robust definition of the specific dietary exclusions being examined.

Andrew S. Day

andrew.day@otago.ac.nz

1 Department of Nutrition and Dietetics, Christchurch Hospital, Christchurch, New Zealand

2 Department of Pædiatrics, Christchurch Hospital, Christchurch, New Zealand

3 Department of Pædiatrics, University of Otago, Christchurch, Christchurch, New Zealand
In the current issue of Digestive Diseases and Sciences, Soresi et al. [7] present their novel work outlining the patterns of gynæcologic disorders in Italian women with and without NCWS. The patients with NCWS in this study were well-characterized following a strict and standardized protocol involving a DBPC wheat challenge. The authors compared symptoms to three control age-matched groups of women recruited at the same gastroenterology unit, including patients with irritable bowel syndrome (IBS) unresponsive to dietary changes, patients diagnosed histologically with $\mathrm{CD}$, and healthy controls without GI symptoms or known disease. The subjects with IBS and the healthy control group had serological tests (but not small bowel biopsies) in order to exclude $\mathrm{CD}$. Furthermore, those with IBS and CD were managed with a short trial of a GFD or ongoing GFD, respectively; the healthy control group did not undergo any dietary changes. Although unlikely, these points raise the possibility of possible confounding factors within the control groups.

More of the subjects with NCWS reported gynæcologic symptoms-particularly menstrual cycle abnormalities, recurrent vaginitis, dyspareunia, and recurrent cystitiscompared with women in the three control groups [7]. Approximately half of the subjects with NCWS with menstrual disorders and a third of those with recurrent vaginitis had symptom resolution after exclusion of dietary wheat. It was not clear, however, if symptoms changed after exclusion of dietary gluten in the control women with $\mathrm{CD}$ or those with IBS.

The entity known as NCWS is reported to manifest with a variety of symptoms, especially neurological symptoms, with numerous GI symptoms. Furthermore, these GI symptoms overlap extensively with symptoms reported by individuals diagnosed with $\mathrm{CD}$ and functional disorders such as IBS. In a recent series of 543 Brazilian patients with NCGS, $93 \%$ reported bloating, $75 \%$ abdominal pain, while $45 \%$ reported diarrhea [8]. An earlier Italian study reported similar symptom patterns, with bloat in $87 \%$, pain in $83 \%$ and diarrhea in 54\% [9]. Eight-one percent of the cohort in the current study had initially come to medical attention 
with GI symptoms, with half of them having diarrhea [7]. The proportion with bloat and pain were not detailed. Nonetheless, the women in the current study appeared to have broadly similar presentations to these previously reported series of patients.

As mentioned in this study, the exact pathogenic and pathogenetic mechanisms leading to NCWS have not been fully elucidated. Nevertheless, innate immune responses may be contributory; for example, a recent report provides preliminary data suggesting differential gene expression profiles in a small group of subjects with NCWS compared with controls [10]. The connection between the intestinal responses seen in NCWS and gynæcologic symptoms is also unclear. Soresi et al. [7] refer to their previous findings of rectal inflammatory changes in individuals with NCWS, proposing pelvic cross-sensitization. The current study, however, did not provide any additional support for this or any other hypothesis tying together NCWS and the increased rate of gynæcologic symptoms.

In the current study, the authors reported that there were not any factors (GI or other) that predicted the subset with gynæcologic symptoms. Nor did they identify any factors that appeared to predict the response to exclusion of wheat in this group. While all of the women with NCWS had resolution of their GI symptoms with exclusion of wheat, only a proportion of those with concurrent gynæcologic symptoms had resolution of those symptoms as well. Clearly being able to elucidate these factors would be of great advantage clinically, but may also provide further clues as to the pathogenesis of these conditions. The inclusion of larger numbers of patients may be required to better clarify these predictive factors.

One aspect that was not considered in the current study is potential contribution of the microbiota. Given the association of the composition of the intestinal microbiota with gastrointestinal disease, including $\mathrm{CD}$, and other literature associating gynæcologic disease with alterations of the vaginal microflora [11], ascertainment of the bacterial patterns may provide etiologic clues. Several reports indicate microbial compositional variations between patients with $\mathrm{CD}$ and those with NCGS, as well as variant microbial responses to commencement of a GFD [1,4]. It would be intriguing to establish whether or not there are any bacterial patterns that associate the co-occurrence of NCWS with gynæcologic symptoms.

In conclusion, this report raises interesting and important findings: that a higher proportion of women with NCWS had gynæcologic symptoms than were observed in women without NCWS, and that some of these women have symptom relief after exclusion of dietary wheat. Nonetheless, there do remain a number of unanswered questions arising from this work. In particular, do women who do not have NCWS with these gynæcologic symptoms also have improvements with wheat exclusion, what are the connections between the GI and pelvic symptoms, and how can one predict those who might respond best to wheat exclusion?

Additional prospective, well-designed and carefully controlled studies are clearly required to confirm these findings in different locations and furthermore to establish generalizability. Such studies should stringently define the population with NCWS using currently agreed-upon guidelines, with careful exclusion of all relevant confounding factors, and focus on further advancing our understanding of these problems.

\section{Compliance with Ethical Standards}

Conflict of interest The authors declare that they have no conflict of interest.

\section{References}

1. Lebwohl B, Sanders DS, Green PHR. Coeliac disease. Lancet. 2018;391:70-81.

2. Choung RS, Unalp-Arida A, Ruhl CE, et al. Less Hidden celiac disease but increased gluten avoidance without a diagnosis in the United States: findings from the National Health and Nutrition Examination Surveys from 2009 to 2014. Mayo Clin Proc. 2016. [Epub ahead of print]. pii: S0025-6196(16)30634-6. https://doi. org/10.1016/j.mayocp.2016.10.012.

3. Food Formulation Trends: Ingredients Consumers Avoid: Market Research Report, 2016. https://www.reportlinker.com/p02051931/ Food-Formulation-Trends-Ingredients-Consumers-Avoid.html. Accessed 2 April 2020.

4. Khan A, Suarez MG, Murray JA. Nonceliac gluten and wheat sensitivity. Clin Gastroenterol Hepatol. 2019. https://doi.org/10.1016/j. cgh.2019.04.009.

5. Catassi C, Elli L, Bonaz B, et al. Diagnosis of non-celiac gluten sensitivity (NCGS): the Salerno experts' criteria. Nutrients. 2015;7:4966-4977.

6. Skodje GI, Sarna VK, Minelle IH, et al. Fructan, rather than gluten, induces symptoms in patients with self-reported non-celiac gluten sensitivity. Gastroenterology. 2018;154:529-539.e2.

7. Soresi M, Incandela S, Mansueto P, et al. Gynæcologic disorders in patients with non-celiac wheat sensitivity. Dig Dis Sci. (Epub ahead of print). https://doi.org/10.1007/s10620-020-06184-8.

8. Gadelha de Mattos YA, Puppin Zandonadi R, Gandolfi L, et al. Self-reported non-celiac gluten sensitivity in Brazil: translation, cultural adaptation, and validation of Italian questionnaire. Nutrients. 2019;11:781.

9. Volta U, Bardella MT, Calabro A, et al. An Italian prospective multicenter study on patients suspected of having non-celiac gluten sensitivity. BMC Med. 2014;12:85.

10. Clemente E, Efthymakis K, Carletti E, et al. An explorative study identifies miRNA signatures for the diagnosis of non-celiac wheat sensitivity. PLoS ONE. 2019;14:e0226478.

11. Greenbaum S, Greenbaum G, Moran-Gilad J, et al. Ecological dynamics of the vaginal microbiome in relation to health and disease. Am J Obstet Gynecol. 2019;220:324-335.

Publisher's Note Springer Nature remains neutral with regard to jurisdictional claims in published maps and institutional affiliations. 